
A. Bressoud
O. Real del Sarte
S. Stiefel
P. Mordasini
L. Perey
J. Bauer
P. F. Leyvraz
S. Leyvraz

\section{Impact of family structure on long-term survivors of osteosarcoma}

Received: 28 June 2006

Accepted: 8 November 2006

Published online: 5 January 2007

(C) Springer-Verlag 2007

A. Bressoud

Department of Clinical Oncology,

Hôpital Pourtalès,

Neuchâtel, Switzerland

O. Real del Sarte $\cdot$ S. Stiefel

Department of Psychiatry,

University Hospital Center,

Lausanne, Switzerland

P. Mordasini · P. F. Leyvraz

Department of Orthopedics,

University Hospital Center,

Lausanne, Switzerland

L. Perey $\cdot$ J. Bauer $\cdot$ S. Leyvraz

Department of Clinical Oncology,

University Hospital Center,

Lausanne, Switzerland

S. Leyvraz $(\bowtie)$

Centre Pluridiciplinaire d'Oncologie,

University Hospital Center (CHUV-06),

1011 Lausanne, Switzerland

e-mail: serge.leyvraz@chuv.hospvd.ch

Tel.: +41-21-3140150

Fax: +41-21-3140181

\begin{abstract}
Goals of work: Long-term outcomes of osteosarcoma have dramatically improved with the use of modern combination therapies. Such aggressive treatments, however, entail chronic complications. In the present study, we assessed the functional, psychological, and familial status of long-term survivors of osteosarcoma treated at our institution. Materials and methods: Fifteen long-term survivors of osteosarcoma were evaluated for functional and psychological sequelae. Functional assessment was based on a method described by Enneking et al. Psychological assessment was based on General Health Questionnaire 28, Inventory Scale for Traumatic Neurosis, and Family System Test. Main results: Ten patients showed mild functional impairments; only five patients were handicapped more seriously. Depressive symptoms were diagnosed in four patients. A total of six patients revealed unbalanced family struc-
\end{abstract}

tures, including three of the four patients with depressive symptoms, all four patients with symptoms of posttraumatic stress disorder, and five of seven patients who showed poor emotional acceptance. Conclusions: Osteosarcoma survivors will generally recover good functional performance. Only a minority of them remain seriously impaired. One third of the patients present depressive symptoms and posttraumatic stress disorder. Poor coping is closely associated with unbalanced family structures. Therefore, the psychological and familial situation of patients with newly diagnosed osteosarcoma should be carefully assessed.

Keywords Osteosarcoma . Functional outcome $\cdot$ Posttraumatic stress disorder · Depression · Family structure

\section{Introduction}

Osteosarcoma develops most commonly in the adolescent phase of extensive bone growth between the ages of 10 and 20 years. Surgical treatment as monotherapy has been shown to yield a 5-year survival of 15-20\%. The 1970 s saw two major shifts in therapy: limb-sparing surgery gradually replaced amputation in selected cases, and postoperative chemotherapy was introduced. More recently, preoperative chemotherapy has been used as well, rendering the surgical treatment modalities less disabling and allowing for osseous prostheses that are better optimized for patient needs. Long-term outcomes have dramatically improved, thanks to this modern combined approach: the 5-year survival of patients with non-metastatic osteosarcoma has increased to $60-75 \%[1,3]$.

Aggressive treatment of cancer is associated with acute and chronic complications that may lead to handicaps interfering with the quality of life and psychological outcome $[16,18]$. Posttraumatic stress disorder was first identified as a phenomenon affecting people with previous exposure to war. It is characterized by a set of symptoms 
emerging several months or years after the traumatic experience. Symptoms may include events being "replayed" in the patient's mind, persistent fear, and physical fatigue. Furthermore, many of these patients avoid people with similar experiences.

These symptoms are not, however, confined to warrelated events. In fact, they are particularly common among cancer survivors. These patients are well aware of the traumatic nature of their disease $[12,26]$. They are faced with existential uncertainties once cancer has been diagnosed. The treatment modalities to which they are subjected may include invasive procedures, severe side effects, and frequent hospitalization, which adds substantially to the psychological burden of the disease as such. Physical sequelae after treatment, such as functional impairments, cognitive alterations, or infertility, may bring up traumatic recollections.

The relationship between physical and psychological sequelae is an important issue even if functional handicaps are not associated with psychosocial maladjustment. Coping with disease has fundamental advantages by promoting social integration [5]. Psychological sequelae and functional performance have been extensively analyzed in survivors of childhood cancer [9, 14, 29]. Some studies focused specifically on long-term survivors after combined treatment regimens for osteosarcoma, but the relationship between family structure and psychosocial outcome has hardly received the attention it deserves. Long-term psychosocial implications of treatment were addressed in the Boston study, which included the largest series of 89 osteosarcoma survivors on record [11]. Two more studies focused on the differences between limb-sparing surgery and amputation in terms of either psychological or functional outcome $[24,30]$. In addition, the patient's family may be an asset or a liability in coping with disease and treatment. The relationship between family structure and psychological sequelae offers significant insight into the social development of young cancer survivors and their perception of physical impairments. With these considerations in mind, we performed the present study to assess the functional, psychological, and familial status of long-term survivors with osteosarcoma treated at our institution by intensive chemotherapy and surgery.

\section{Materials and methods}

\section{Patient selection}

In 1983-1994, a total of 39 patients with osteosarcoma were treated at various departments (multidisciplinary oncology, pediatric oncology, and orthopedics and traumatology) of the University Hospital Center (CHUV) in Lausanne, Switzerland [20]. Five patients had extraosseous osteosarcomas and were treated by surgical resection and radiotherapy. Of the other 34 patients with conventional osteosarcoma, 3 did not survive after suboptimal chemotherapy, 2 survived after surgical resection without adjuvant chemotherapy, and 3 survived after surgical resection combined with postoperative chemotherapy. A total of 26 patients were treated by intensive chemotherapy both before and/or after surgery. Fifteen of the long-term survivor patients agreed to participate in the study. The study was accepted by the independent medical committee of the oncological department, and the patients gave an oral informed consent according to the rules of the ethical committee.

\section{Patient characteristics}

A total of 15 long-term survivors ( 7 women and 8 men) were followed up and evaluated for their functional impairments and psychological sequelae. Their mean age at the time of evaluation was $26(19-47)$ years. The mean interval between diagnosis and evaluation was $11(2.5-19)$ years. The tumors encompassed femoral $(n=8)$, tibial $(n=2)$, proximal humeral $(n=3)$, pelvic $(n=1)$, and metatarsal $(n=1)$ locations. Surgical treatment consisted in resection $(n=8)$ or amputation $(n=7)$. In ten patients, adjuvant chemotherapy was applied both before and after surgery $(\operatorname{COSS} 82, n=5$; $\operatorname{COSS} 86, n=4$; Rosen T-4, $n=1)$. In three patients, chemotherapy was only applied in the postoperative period (Rosen T-4, $n=2$; Rosen T-10, $n=1$ ). The remaining two patients did not receive chemotherapy. Conventional osteosarcoma was histologically present in 13 patients. The remaining two patients had juxtacortical osteosarcoma but were also included because they revealed similar patterns of motor impairment after surgical treatment. Pertinent patient data are summarized in Table 1.

\section{Data collection}

All functional data for the 15 long-term survivors were collected by a single orthopedic surgeon (M.P.). The psychological evaluation had a twofold purpose: (1) identification of depressive features and posttraumatic stress disorder and (2) determination of family structures and their association with psychosocial and functional outcomes. Likewise, all psychological data were collected in a very uniform fashion by the same psychologist (R.O.). The surgeon and the psychologist were blinded to each other's results. All patients were treated and followed up in the same center within a relatively short period of time. Hence, the study conditions were very homogeneous. Our study was observational without pre-planned statistical analysis. 
Table 1 Patient characteristics

\begin{tabular}{|c|c|c|c|c|c|c|c|c|}
\hline Patient & Sex & Born & Age & Site & Type of surgery & $\begin{array}{l}\text { Preoperative } \\
\text { chemotherapy }\end{array}$ & $\begin{array}{l}\text { Postoperative } \\
\text { chemotherapy }\end{array}$ & Protocol \\
\hline 1 & M & 1970 & 15 & Distal femur & Resection & Yes & Yes & COSS 82 \\
\hline 2 & $\mathrm{~F}$ & 1952 & 33 & Distal femur & Resection & No & No & \\
\hline 3 & $\mathrm{M}$ & 1972 & 16 & Distal femur & Resection & Yes & Yes & COSS 86 \\
\hline 4 & $\mathrm{~F}$ & 1977 & 16 & Humerus & Resection & Yes & Yes & COSS 86 \\
\hline 5 & $\mathrm{~F}$ & 1965 & 19 & Humerus & Resection & Yes & Yes & COSS 82 \\
\hline 6 & $\mathrm{M}$ & 1973 & 10 & Distal femur & Amputation & Yes & Yes & COSS 82 \\
\hline 7 & M & 1949 & 34 & Humerus & Resection & No & No & \\
\hline 8 & M & 1974 & 9 & Proximal tibia & Amputation & Yes & Yes & COSS 82 \\
\hline 9 & $\mathrm{~F}$ & 1966 & 28 & Pelvis & Resection & Yes & Yes & COSS 86 \\
\hline 10 & M & 1964 & 12 & Distal femur & Disarticulation & Yes & Yes & RosenT4 \\
\hline 11 & $\mathrm{~F}$ & 1969 & 16 & Metatarsus & Amputation & Yes & Yes & COSS 82 \\
\hline 12 & $\mathrm{~F}$ & 1977 & 12 & Distal femur & Amputation & No & Yes & RosenT4 \\
\hline 13 & M & 1966 & 21 & Distal femur & Amputation & No & Yes & RosenT4 \\
\hline 14 & $\mathrm{~F}$ & 1975 & 13 & Distal femur & Resection & No & Yes & RosenT10 \\
\hline 15 & M & 1967 & 20 & Proximal tibia & Amputation & Yes & Yes & COSS 86 \\
\hline
\end{tabular}

Functional assessment

Global functional assessment (adapted from Enneking et al. [8]) The test system comprised six functional parameters: pain, motion, strength, stability, emotional acceptance, and complications. Each parameter was numerically rated along a 6-grade scale ranging from 0 to 5. The individual scores were added up to obtain a global functional score that was assessed as a percentage of the maximum score.

Objective functional assessment For the purpose of this study, the global scores were additionally broken up into objective vs subjective parameters. The objective parameters included motion, strength, stability, and complications. These scores were added up to obtain an objective functional score that was assessed as a percentage of the maximum score.

Subjective functional assessment The subjective parameters included in the global functional assessment were pain and emotional acceptance. These scores were added up to obtain a subjective functional score that was assessed as a percentage of the maximum score.

The difference between the objective and the subjective assessments was calculated for each patient and defined as significant when it was $>25 \%$ (Table 3 ).

\section{Psychological assessment}

General health questionnaire 28 This questionnaire has been designed as a community-screening tool to determine nonspecific psychiatric morbidity. It comprises four sec- tions: somatic symptoms, anxiety and insomnia, social dysfunction, and depression. Each of these parameters includes seven subitems. In this way, the investigator obtains four subscores, which are added up to obtain a global score. Each subitem has two possible answers that are considered positive and two possible answers that are considered negative. Each positive reply scores 1 point. Thus, the maximum global score would be 28 points ( 7 points in each section). Global scores $>5$ are considered positive for depressive symptoms [4].

Inventory scale for traumatic neurosis This tool to detect symptoms of posttraumatic stress disorder has been described and validated by Steinitz and Crocq [27] and is presently only available in French. It comprises a total of 46 items in five sections to assess the intensity of traumatic sequelae. A score of $0-5$ is obtained for each section, thus the maximum total score is 25 . Based on this total score, traumatism is classified as mild (0-10), moderate (11-15), severe (15-20), or very severe (21-25).

Family system test This test system is used to evaluate intrafamilial cohesion and hierarchy by spatial arrangement of figures on a board [10, 21, 22]. Patients were instructed to arrange schematic figurines $(8 \mathrm{~cm}$ high) representing female and male individuals across a board $(45 \times 45 \mathrm{~cm})$ divided into 81 squares $(5 \times 5 \mathrm{~cm}$ each $)$ to indicate cohesion within the family. They were also shown how these figurines could be elevated using cylindrical blocks of three sizes $(1.5,3$, and $4.5 \mathrm{~cm})$ in any number and combination to indicate hierarchical relationships within the family. The investigator recorded the locations and heights of the various figurines as arranged by the patient. The recorded configurations formed the basis for 
cohesion and hierarchy scores. Hierarchy was estimated by the difference between the less influential parent and the most influential child. It was classified as low (difference of less than one small block), medium (one small or middle-sized block), or high (one large block or more). Cohesion was estimated by the proximity of the figurines on the board. The cohesion was classified as high (all figurines adjacent), medium (all figurines inside a space of $3 \times 3$ squares), or low.

The patient sample was characterized by heterogeneous family circumstances including both married individuals with or without children $(n=4)$ and unmarried $(n=11)$ individuals. For married patients, the nuclear family including spouses and children was used as reference, and for unmarried patients, it was related to the family they lived with at the time of diagnosis and treatment.

Based on the configuration obtained, the family structure was classified as balanced or unbalanced. Configurations characterized by medium/strong cohesion and medium hierarchy were considered to reflect a balanced family structure. All other configurations (medium cohesion and strong hierarchy, low cohesion and medium hierarchy, or any extreme values on both ends of the spectrum) were considered to reflect an unbalanced family structure.

\section{Results}

The functional and psychological data collected in the present study are summarized in Table 2 .
Functional assessment

Global functional assessment The functional performance of our patients was fairly good. We obtained a median global score of 24 (16-30) points, equaling $67 \%$ of the maximum attainable score of 36 points. Interestingly, the functional scores obtained in five of the six amputated patients were higher than the average score for the entire sample.

Objective functional assessment Motion, strength, and stability were separately analyzed as objective functional parameters. The median score based on these objective parameters was 17 points, equaling $71 \%$ of the maximum attainable score of 24 points. Overall, the objective performance as measured by the orthopedic surgeon was markedly better than the subjective performance reported by the patients themselves (see below). Despite having undergone extensive surgery, only five patients (numbers 3 , $4,6,10$, and 14) revealed serious impairments (score $\leq 14$ ), including hip disarticulation $(n=1)$, leg amputation $(n=1)$, knee arthrodesis $(n=2)$, and humeral allograft $(n=1)$.

Subjective functional assessment Pain and emotional acceptance were separately analyzed as subjective functional parameters. The median score based on these subjective parameters was 7 points, equaling $60 \%$ of the maximum attainable score of 12 points. Four patients reported no pain; ten patients reported mild pain. Only one patient reported severe pain.

Table 2 Functional and psychological results

\begin{tabular}{|c|c|c|c|c|c|c|}
\hline & Functional & & & Psychological & & \\
\hline Patient & Objective $(\max =24)$ & Subjective $(\max =12)$ & Total $(\max =36)$ & GHQ28 & PTSD & FAST \\
\hline 1 & 18 & 6 & 24 & Negative (2) & Mild (0) & Balanced \\
\hline $2^{\mathrm{a}}$ & 16 & 2 & 18 & Positive (18) & Mild (5) & Unbalanced \\
\hline 3 & 14 & 8 & 22 & Negative (0) & Mild (0) & Balanced \\
\hline 4 & 14 & 8 & 22 & Negative (0) & Mild (3) & Balanced \\
\hline $5^{\mathrm{a}}$ & 20 & 8 & 28 & Positive (13) & Mild (3) & Balanced \\
\hline 6 & 10 & 8 & 18 & Negative (2) & Mild (0) & Balanced \\
\hline $7^{\mathrm{a}}$ & 16 & 10 & 26 & Negative (4) & Mild (3) & Balanced \\
\hline 8 & 24 & 8 & 32 & Negative (2) & Mild (4) & Unbalanced \\
\hline $9^{\mathrm{a}}$ & 22 & 8 & 30 & Negative (1) & Mild (3) & Balanced \\
\hline 10 & 10 & 6 & 16 & Negative (1) & Mild (2) & Balanced \\
\hline 11 & 22 & 6 & 30 & Negative $(0)$ & Mild (5) & Unbalanced \\
\hline 12 & 20 & 6 & 26 & Positive (0) & Mild (2) & Unbalanced \\
\hline 13 & 18 & 6 & 24 & Negative (4) & Mild (2) & Unbalanced \\
\hline 14 & 14 & 6 & 20 & Negative (2) & Mild (1) & Balanced \\
\hline 15 & 20 & 10 & 30 & Positive (19) & Mild (5) & Unbalanced \\
\hline Median & 17 & 7 & 24 & & & \\
\hline
\end{tabular}

GHQ28 General Health Questionnaire 28, PTSD posttraumatic stress disorder, FAST family system test ${ }^{\mathrm{a}}$ Married 
The overall discrepancy between subjective and objective performance arose from seven patients (numbers 1, 2, $8,9,11,12$, and 13) who reported a poorer outcome than appeared justified from their objective performance (difference in percentage of maximum score between objective and subjective assessment $\geq 25 \%$, Table 3 ). Among the 4 married and the 11 unmarried patients, 2 and 5 patients, respectively, complained of a worse personal performance compared to the objective assessment.

Psychological assessment

General health questionnaire Only four patients (numbers $2,5,12$, and 15 ) revealed depressive symptoms, including both two amputated patients and two patients treated by limb-sparing surgery. None of these patients belonged to the subset of five patients who revealed serious functional impairments. Questionnaires positive for depression showed no correlation with poor functional outcomes or with conservative surgery vs amputation. In other words, no association was observed between depressive symptoms on the one hand and type of surgery or functional impairments on the other.

Inventory scale for traumatic neurosis None of the patients revealed a complete syndrome of posttraumatic stress disorder. Four patients, however, did reveal some distinctive symptoms of anxiety and depression (numbers $2,8,11$, and 15). Three of these four patients belonged to the subset whose subjective outcomes were poorer than appeared justified from their objective performance.

Family system test Balanced family structures were identified in nine patients and unbalanced family structures in six patients, of whom one was and five were not married. Five of the seven patients who reported poorer outcomes than seemed justified from their objective performance revealed unbalanced family structures. Conversely, all patients who showed good subjective acceptance revealed balanced family structures. There was only one exception (number 15), but this patient was having a temporary relationship problem at the time. Unbalanced family structures were also identified in all four patients who revealed symptoms of posttraumatic stress disorder and in three of the four patients who returned questionnaires positive for depression. A Wilcoxon rank sum test confirmed that subjective acceptance was significantly lower in patients with an unbalanced familial structure $(p=0.01)$.

\section{Discussion}

To our knowledge, the present study demonstrates, for the first time by formal investigation, that an unbalanced family structure is associated with adverse outcome in osteosarcoma survivors. Seven of the 15 patients investigated were significantly more pessimistic about their functional outcome than appeared justified from their objective performance. This discrepancy indicates that they did not cope too well with their disease and the treatment modalities involved. All these patients but two exhibited unbalanced family structures, which was true in only one of the eight remaining patients. Unbalanced family structures were also identified in most of the patients who revealed depressive symptoms. The marital status might certainly have had an impact, but our cohort was too small to allow any firm conclusions. Indeed, if only 1 from the 4 married patients had an unbalanced familial structure, still 2 of them were complaining of a poor functional outcome, similar to the 5 among the 11 non-married patients.

The functional assessments yielded fairly good results. Overall, the functional performance based on objective parameters reached $72 \%$ of what would be considered normal. Functional impairments were minor in ten patients and major in only five patients, who invariably showed

Table 3 Comparison between functional assessments of patients with balanced or unbalanced familial structure

\begin{tabular}{|c|c|c|c|c|c|c|c|}
\hline \multicolumn{4}{|c|}{ Assessments of patients with a balanced familial structure } & \multicolumn{4}{|c|}{ Assessments of patients with an unbalanced familial structure } \\
\hline $\begin{array}{l}\text { Patient } \\
\text { number }\end{array}$ & $\begin{array}{l}\text { Objective } \\
\text { (\% max score) }\end{array}$ & $\begin{array}{l}\text { Subjective } \\
\text { (\% max score) }\end{array}$ & Difference $(\%)$ & $\begin{array}{l}\text { Patient } \\
\text { number }\end{array}$ & $\begin{array}{l}\text { Objective } \\
\text { (\% max score) }\end{array}$ & $\begin{array}{l}\text { Subjective } \\
\text { (\% max score) }\end{array}$ & Difference $(\%)$ \\
\hline 1 & 75 & 50 & +25 & $2^{\mathrm{a}}$ & 66 & 16 & +50 \\
\hline 3 & 58 & 66 & -8 & 8 & 100 & 66 & +34 \\
\hline 4 & 58 & 66 & -8 & 11 & 91 & 50 & +41 \\
\hline $5^{\mathrm{a}}$ & 83 & 66 & +7 & 12 & 83 & 50 & +33 \\
\hline 6 & 41 & 66 & -5 & 13 & 75 & 50 & +25 \\
\hline $7^{\mathrm{a}}$ & 66 & 83 & -7 & 15 & 83 & 83 & 0 \\
\hline $9^{\mathrm{a}}$ & 91 & 66 & +25 & & & & \\
\hline 10 & 41 & 50 & -9 & & & & \\
\hline 14 & 58 & 50 & +8 & & & & \\
\hline
\end{tabular}

${ }^{\mathrm{a}}$ Married 
good emotional acceptance. Furthermore, good functional scores were obtained in five of the six amputated patients. In the majority of cases, pain was not an issue.

Our results are consistent with findings in the literature that most patients adjust well to functional impairments and that psychological or functional advantages of limbsparing over amputational surgery cannot be demonstrated $[11,19,24,30]$. Based on a large series, Greenberg et al. [11] reported that pain was absent or mild in $79 \%$ of patients and that $80 \%$ were able to resume their previous activities. Babin et al. [2] reported that one third of 20 patients with lower-limb osteosarcoma recovered well from a functional viewpoint. With $68 \%$ of normal function, their long-term results were similar to the ones observed in the present study.

Several risk factors for adverse outcome in cancer survivors have been reported. These include low education, low income, sex-specific factors, or functional limitations $[13,17,32,33]$. The ability to cope with chronic disease is paramount [15]. Patients who stand up to the challenges of cancer and the treatment modalities involved will achieve better psychosocial integration [21].

Our result that one third of patients revealed unbalanced family structures and coped poorly with disease is consistent with a study by Boman and Bodegard [5], who reported poor coping in $27 \%$ of 30 childhood cancer survivors. Osteosarcoma often occurs in adolescents and young adults who are going through an important period of life marked by the transition from a centripetal phase of family closeness to a centrifugal phase of family disengagement [23]. The quest for private and professional autonomy poses major challenges. The family is instrumental in this phase by balancing the needs of its various members for cohesion and hierarchy. Cancer can seriously interfere with the natural development occurring in this phase, such that the young patient's quest for autonomy may be slowed down. In this situation, the family may lose its balancing function by strengthening or weakening cohesion and hierarchy in a way that is counterproductive. A family structure that is out of balance may leave the patient unable to meet the challenges of adulthood. Consequently, the patient may develop depressive symptoms later in life or project his psychological maladjustment on the functional impairment. Therefore, prompt intervention with the aim to rebalance the family structure can be greatly conducive to psychological development and improve functional outcomes [22].

As the survival rates of childhood cancer increased after the introduction of intensive chemotherapy and multidisciplinary approaches, various studies on psychological sequelae were performed. The available reports focused on different patient groups using a variety of methods that cannot be readily compared. Some authors observed good psychological outcomes $[14,16,29]$. Others reported that a significant minority of young survivors had problems adjusting and revealed depressive symptoms $[5,9]$. In a recent study, $20 \%$ of young adult survivors of childhood cancer (mainly leukemia, lymphoma, and sarcoma) met criteria for posttraumatic stress disorder [12].

In a large quality-of-life survey of osteosarcoma survivors, Greenberg et al. [11] reported depressive symptoms in $12 \%$ and posttraumatic stress disorder in $13 \%$ of patients. Some symptoms of posttraumatic stress disorder were also noted in four patients of our sample, although none of these cases revealed the syndrome as such. Thus, our results are more in accordance with another large series of cancer survivors, in which up to one fifth of patients showed symptoms of posttraumatic stress syndrome $[12,28]$.

Several authors have singled out the patient's fighting spirit as an important prognostic factor [31]. Disease prognosis clearly deteriorates when anxiety and depression are present. The General Health Questionnaire 28, which was used in this study, focuses on coping mechanisms and is therefore an important prognostic tool. Roughly one quarter of our patients $(n=4)$ returned questionnaires that were positive for depression, which compared quite favorably with a rate of $>40 \%$ obtained in a large sample of patients attending general practitioners [6]. This confirms the finding of a previous study that depression does not seem to be a major problem in osteosarcoma survivors [7].

Although our sample was not large enough for in-depth statistical analysis, a number of conclusions can nevertheless be drawn: (1) osteosarcoma survivors tend to recover much of their functional performance, as only a minority of our patients were seriously handicapped; (2) symptoms of depression and posttraumatic stress disorder are observed in around one third of our patients; (3) most patients with psychological symptoms are characterized by unbalanced family structures, although this is rarely the case in patients with good mental disposition.

To summarize, there seems to be a strong correlation between psychological well-being and family structure in osteosarcoma survivors. Patients with unbalanced family structures are overly pessimistic about their functional performance, which puts them at a high risk for depression. It is very important to identify such patients. Therefore, all patients with newly diagnosed osteosarcoma should be carefully assessed for their psychological and familial situation as an integral part of pretherapeutic management.

All things considered, it is reasonable to assume that timely intervention can significantly improve the success of psychological, social, and occupational rehabilitation after successful treatment of osteosarcoma. Cure is more than eradication of cancer [25]. 


\section{References}

1. Aksnes LH, Hall KS, Folleraas G, Stenwig AE, Bjerkehagen B, Taksdal I, Winderen M, Bruland OS, Saeter G (2006) Management of high-grade bone sarcomas over two decades: the Norwegian Radium Hospital experience. Acta Oncol 45(1):38-46

2. Babin SR, Simon P, Babin-Boilletot A, Bellocq JP, Marcellin L, Dosch JC (1996) Complications and results in high-grade osteosarcoma of the lower limb. A study of 20 cases. Rev Chir Orthop 82:14-21

3. Bielack SS, Kempf-Bielack B, Delling G, Exner U, Flege S, Helmke K, Katz R, Werner M, Winkelmann W, Zoubek A, Jurgens H (2002) Prognostic factors in high-grade osteosarcoma of the extremities or trunk: an analysis of 1,702 patients treated on neoadjuvant cooperative osteosarcoma study group protocols. J Clin Oncol 20:776-790

4. Bolognini M, Bettschart W, ZehnderGubler M, Rossier L (1989) The validity of the French version of the GHQ-28 and PSYDIS in a community sample of 20 year olds in Switzerland. Eur Arch Psychiatr Neurol Sci 238(3): 161-168

5. Boman K, Bodegard G (1995) Psychological long-term coping with experience of treatment in childhood cancer survivors. Acta Paediatr 84:1395-1402

6. Bordmann AP (1987) The general health questionnaire and the detection of emotional disorder by general practitioners, a replicated study. Br J Psychiatry 151:373-381

7. Cameron KT (1993) Psychological effects of amputation in osteosarcoma. In: Osteosarcoma in adolescents and young adults. Kluwer, Boston, pp 39-44

8. Enneking WF, DunHam W, Gebhardt MC, Malawar M, Pritchard DJ (1993) A system for functional evaluation of reconstructive procedures after surgical treatment of tumors of the musculoskeletal system. Clin Orthop Relat Res 286:241-246

9. Fritz GK, Williams JR, Amylon M (1988) Psychosocial sequelae in pediatric cancer survivors. Am J Orthopsychiatr 58(4):552-561
10. Gehring T. Family System Test (FAST), Authorized English translation by Anita Arone Reitzle, Zurich, Switzerland. First publication in 1993 By Beltz Verlag, Germany, under the title of Familiensystemtest (FAST). Copyright Beltz Gessellschaft

11. Greenberg DB, Goorin A, Gebhardt MC, Gupta L, Stier N, Harmon D, Mankin H (1994) Quality of life in osteosarcoma survivors. Oncology 8(11):19-25

12. Hobbie WL, Stuber M, Meeske K, Wissler K, Rourke M, Ruccione K, Hinkle A, Kazak AE (2000) Symptoms of posttraumatic stress in young adult survivors of childhood cancer. J Clin Oncol 18:4060-4066

13. Hudson MM, Mertens AC, Yasui Y, Hobbie W, Chen H, Gurney JG, Yeazel M, Recklitis CJ, Marina N, Robinson L, Oeffinger K (2003) Health status of adult long-term survivors of childhood cancer. JAMA 290:1583-1592

14. Kazak AE, Meadows AT (1989) Families of young adolescents who have survived cancer: socio-emotional adjustment, adaptability and social support. J Pediatr Psychol 14:175-191

15. Koopmann HM, Koetsier JA, Taminiau AH, Hijnen KE, Bresters D, Egeler RM (2005) Health-related quality of life and coping strategies of children after treatment of a malignant bone tumor: a 5-year follow-up study. Pediatr Blood Cancer 45:694-699

16. Maunsell E, Pogany L, Barrera M, Shaw AK, Speechley KN (2006) Quality of life among long-term adolescent and adult survivors of childhood cancer. J Clin Oncol 24:2527-2535

17. Nagarajan R, Neglia JP, Clohisy DR, Yasui Y, Greenberg M, Hudson M, Zevon M, Tersak JM, Albin A, Robinson LL (2003) Education, employment, insurance, and marital status among 694 survivors of pediatric lower extremity bone tumors. Cancer 97:2554-2564

18. Nicholson HC, Mulvihill JJ (1993) Late effects of therapy in survivors of childhood and adolescent osteosarcoma. Cancer Treat Res 62:45-48

19. Pardasaney PK, Sullivan PE, Portney LG, Mankin HJ (2006) Advantage of limb salvage over amputation for proximal lower extremity tumors. Clin Orthop Relat Res 444:201-208

20. Perey L, Guillou L, Jallut D, Beck D, Livio JJ, Leyvraz S (1995) Traitement combiné des ostéosarcomes: expérience lausannoise. Méd et Hyg 53:23182324
21. Real del Sarte O (1996) Psychosomatique et chronicité débat autour d'une recherche. Thér Fam 17:225-239

22. Real del Sarte O, Stiefel F, Leyvraz S, Bauer J, Gehring TM, Guex P (1998) The Family System Test, a pilot study in families with a young adult member with cancer. Support Care Cancer 6:416-420

23. Rolland JS (1987) Chronic illness and the life cycle. Fam Proc 26:203-221

24. Rougraff BT, Simon MA, Kneisl JS, Greenberg DB, Mankin HJ (1994) Limb salvage compared with amputation for osteosarcoma of the distal end of the femur. J Bone Jt Surg 76-A:649656

25. Schwartz CL (2003) Health status of childhood cancer survivors. Cure is more than the eradication of cancer. JAMA 290:1641-1643

26. Schwartz L, Drotar D (2006) Posttraumatic stress and related impairment in survivors of childhood cancer in early adulthood compared to healthy peers. J Pediatr Psychol 31:356-366

27. Steinitz A, Crocq L (1992) L'inventaire-échelle de névrose traumatique. Psychol Méd 24:437-445

28. Taieb O, Moro MR, Baubet T, RevahLevy A, Flament MF (2003) Posttraumatic stress symptoms after childhood cancer. Eur Child Adolesc Psychiatry 12:255-264

29. Wasserman AL, Thompson EI, Williams JA, Fairclough DL (1987) The psychological status of survivors of childhood/adolescent Hodgkin's disease. Am J Dis Child 141(6):626-631

30. Weddington WW Jr, Segraves KB, Simon MA (1985) Psychological outcome of extremity sarcoma survivors undergoing amputation or limb salvage. J Clin Oncol 3:1393-1399

31. Wood B, Watkins JB, Boyle JT (1989) The psychosomatic family model, an empirical and theoretical analysis. Fam Proc 28:399-417

32. Zebrack BJ, Zeltzer LK, Whitton J, Mertens AC, Odom L, Berkow R, Robinson LL (2002) Psychological outcomes in long-term survivors of childhood leukaemia, Hodgkin's disease, and non-Hodgkin's lymphoma. Pediatrics 110:42-52

33. Zebrack BJ, Zevon MA, Turk N, Nagarajan R, Whitton J, Robinson LL, Zeltzer LK (2006) Psychological distress in long-term survivors of solid tumors diagnosed in childhood: a report from the childhood cancer survivor study. Pediatr Blood Cancer, Jun 5; [Epub ahead of print] 